\title{
A Fuzzy Scheduling Controller For The Computer Disk File Track-Following Servo
}

\author{
Jis-Yush Yen; Fu-Jeng Wangł Yung-Yaw Chen ${ }^{\ddagger}$
}

\begin{abstract}
In this paper, a fuzzy tuning algorithm is developed for the computer disk drive track following servo system. A Zentek 3100 disk drive is modifled, and a controller scheduling capability is added to the servo loop to compensate for the plant variations as the actuator is locked on to different tracks. The mathematical models for the actuator on a number of tracks chosen are experimentally identified. The $H_{\infty}$ design technique is then employed to obtain a robust optimal controller for each operating point. A combined controller is then calculated using a fuzzy algorithm. The fuszy algorithm is used to represent the complex relationship between the track number and the corresponding controller. It is shown that with the controller scheduling action, the closed-loop performance is improved for the actuator at every track positions.
\end{abstract}

\section{INTRODUCTION}

One of the most popular research topics addressed by the control engineers nowadays is the use of "fuzzy logic con-

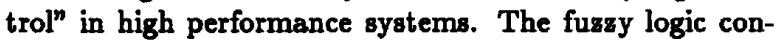
troller is based upon linguistic rules which translate human thinking into machine codes. It is thus very suitable for applications where the system characteristics or the control actions are hard to be expressed by mathematical expressions. The fuszy controller is known to have very nice performances such as fast settling time and performance robustness against plant variations ( $\mathrm{Li}$ and Lau, 1989, Peng and Liu, 1988). Many successful applications have also been reported (Sugeno, 1985), and new systems are seeking to use this technology. While the fuxzy controller is gaining its popularity, it is noted that the furzy logic operation involves very complicated calculation. Therefore, before fast furgy logic CPUs are available, it is not suitable for direct implementation in high speed, high bandwidth systems.

In this paper, a fuzzy tuning algorithm is developed

Associate Profensor, Department of Mechanical Engineering,

I Graduate Student, Department of Mechanical Engineering,

'Aseociate Profeseor, Department of Electrical Engineering, National Taiwan University, Taipei, Taiwan, R.O.C. for the computer disk drive track following servo system. The computer disk drive, often called the hard disk drive (HDD), is the most popular on-line data storage device used in today's computer systems. Over the years, HDD has survived the challenges from the magnetic tape drives, the electronic on-board memory (RAM), the optical disk drives, and has become the fastest and most reliable online massive data storage device used by many computer manufacturers (Bajorek, 1990). Recently, both IBM and Futamoto Hitachi had announced new experimental drives which records more than 1 Giga bits/in ${ }^{2}$ (Wood 1990, Zorpette 1991). This achievement has made the HDD the most promising memory device for future miniaturized palm top or pocket computers.

As the recording density further increases (more than 20,000 tracks/in in the new experimental disk drives), the head positioning servo will now have to position the head to within $0.1 \mu \mathrm{m}$ accuracy (one tenth of the today's accuracy). Many mechanical problems will start to emerge. The stiction of the actuator bearing caused by the manufacturing tolerances will start to affect the achievable servo resolution. The drag force and the bias force introduced by the flexible cable linking the moving part of the drive to the disk drive controller board will also cause dramatic variation in the system behavior. Traditional linear servo will no longer be satisfactory, and some tuning ability is necessary.

In this paper, the track following servo controller of a Zentek 3100 disk drive manufactured by the Zentek Co., Taiwan is modified. The design of the computer disk file servo controller is the result of many year's experiences. Over the years, the design technology has to gradually evolve from simple P-I-D controller to the $H_{\infty}$ optimization technique used in today's disk drive (Commander and Taylor, 1980, Workman, 1987, Hanselmann, 1988, Franklin, et.al., 1990, Yen, et.al., 1991). It is very difficult to just discard all these progresses and design a totally new compensator. Therefore, instead of replacing the traditional linear controller, a controller scheduling capability is implemented so that the plant variations due to different tracks can be compensated. The mathematical model for the actuator on various tracks is experimentally identified. The $H_{\infty}$ design technique is then employed to 
obtain a robust optimal controller for the disk head actuator at each track. The actual controller for the disk drive actuator at various tracks is then calculated form all these controllers using a fussy interpolation. The fussy interpretation is used here mainly for its convenience at representing the relationship among various controllers. The identified track models do not show any convenient correlation with the track position. A simple function to interpolate among the controllers can not be obtained. The fuszy interpolation is therefore the most adequate tool in this situation.

It is shown that, provided with an accurate plant model, the $H_{\infty}$ design procedure for optimal control is very straight forward. The simulation results for each track can be directly implemented. The Schur balanced truncation technique is applied to ensure a low order controller. Without the controller scheduling capability, the quality of the control depends very much on the nominal track which the design is base on. Without careful selection of the nominal track, the servo system can become unstable at some situations. With the controller scheduling action, the servo loop remain stable for all the track positions. Furthermore, the error can be kept at a lower level then the case when only a single controller is used.

\section{SYSTEM IDENTIPICATION}

The disk drive used in this experiment is a Zentek 3100 hard disk drive manufactured by the Zentek Co., ShinChu, Taiwan. The disk drive holds up to 120 megabytes formatted data with an access time of $15 \mathrm{~ms}$. Digital data is recorded as different magnetizations in concentric rings on the surfaces of several spinning disks. An actuator is used to drive the read/write head to access the data. For the Zentek $\mathbf{3 1 0 0}$ disk drives, a rotary type actuator is used. The data tracks are spaced $20 \mu m$ apart on the disk surface, and the servo controller will have to maintain the head to within less then $1 \mu \mathrm{m}$ from the dats track center. The actuator is driven by a voice coil motor. The positioning information in this disk drive is written on a dedicated servo surface and all the heads are assumed to follow the servo head rigidly. For the servo design purpose, the signal entering the voice coil motor current amplifier is defined as the plant input, and the position error signal (PES) measured by the data channel demodulator for the servo surface is defined as the plant output. A linear system model is usually used to describe the system behavior.

The model identification is performed by curve fitting the frequency response of the actuator dynamics. An HP3563A dynamic structural analyzer is used for measuring the frequency response and for performing the curve fit. The signal into the voice coil motor is defined as the plant input, and the PES signal from the demodulator is defined as the plant output. The actuator is running under closed loop control while a sweep sine signal is added to the plant input to excite the plant dynamics. The closedloop identification is necessary for the actuator system is very close to unstable without proper compensation. It is also well known (Ljung 1987) that the persistence excitation condition can atill be met under closed loop control provided the disturbance signal is rich enough. A linear system is assumed so that least square curve fit in the frequency domain can be conducted.

The frequency response of the actuator at track 0 and the response of its curve fit model using a 6 th order model is shown in Fig.1. It is noted that the first resonance peak of the actuator occurs at around $2 K H z$. Higher order system model has been used, and not much detail is obtained. Therefore, a 6th order model is decided to be an adequate choice for the plant. The plant model at tracks $0,300,500,700$, and 992 are identified. The comparison among the frequency responses of the identified models for various tracks are shown in Fig.2. It can be seen that a simple function to describe the relationship between the track number and the low frequency gain can not be easily obtained. The fussy description thus becomes the most effective tool for this occasion. It is also observed that the major changes in the responses occurs in the low frequency region. The resonance frequency, however, remain almost unchanged. Quite contrary to our expectation. Although a full controller interpolation is used in this work, in practice only a change in the low frequency response is necessary.

\section{III. $H_{\infty}$ Desian Of The Computer Disk DRIVE Track Following Controller}

The $H_{\infty}$ optimization technique is used for the controller design. The complementary sensitivity transfer function of the system is denoted by $T(s)$, and the sensitivity transfer function is denoted by $S(s)$. The weighting function for the sensitivity transfer function is $W_{1}(s)$, and the weighting function for the complementary sensitivity transfer function is $W_{3}(s)$.

The control objective is to maintain the head centered within $1 \mu m$ range from the track center. The desired closed-loop bandwidth is defined to be $400 \mathrm{~Hz}$. A $-40 d B /$ decade attenuation beyond the bandwidth is desired for robustness. In order to over come the steady state error, a very low gain lead type weighting function for the complementary sensitivity transfer function is used. The responses of the weighting functions are shown in Fig.3. Notice that the frequency is normalised to $400 \mathrm{H} z$ in these equations. The $H_{\infty}$ calculation for the 6 th order model is conducted to obtain a 12th order compensator. Schur balanced truncation technique is then used to obtain a 6th order controller. The design procedure is carried out for all the plant models identified in the previous section. 
Fig. 4 shows the frequency responses of the resulting compensators for each operation point. All the controllers are basically composed of a very large lag compensator in the low frequency region to provide enough loop gain, and in the high frequency area are basically a lead-lag compensator followed with a sharp high frequency roll-off.

\section{The Fugzy Scheduling Controller Design}

The scheduling algorithm is designed based upon the concept that "the best controller for the system should be used whenever it is available". Since the optimal controller is not available for all the tracks, the desired controller will be obtained by interpolating the nearest neighboring controllers. The actual RMS values for the track following error will be compared in the experiment.

The plant model at the five track positions is denoted by $P_{0}, P_{300}, P_{500}, P_{700}$, and $P_{992}$, and the corresponding $\boldsymbol{H}_{\infty}$ controllers are denoted by $C_{0}, C_{300}, C_{500}, C_{700}$, and $C_{992}$. As mentioned in the beginning of this section, the basic concept for tuning is to used the most proper controller when available. When the best controller is not available, then the controller is obtained by the nearest neighboring controllers. This concept can be achieved with various methods. The most direct method is to use the entire transfer function for the interpolation. This method will produce a resulting controller with an order as high as twice the original controller. In our approach, the controllers are represented with their poles and zeros. It is noted that the poles and seros can be grouped into four different kinds. This is reasonable under the assumption that the plant does not under go abrupt changes moving from the inside of the disk to the outside. Thus, the poles and seros of the corresponding optimal controller will also move gradually as the actuator move tracks.

The controller scheduling algorithm can now be designed. The 6th order controller is defined as

$C(z)=K \cdot \frac{\left(z-z_{1}\right)\left(z-z_{2}\right)\left(z-z_{3}\right)\left(z-z_{4}\right)\left(z-z_{5}\right)\left(z-z_{6}\right)}{\left(z-p_{1}\right)\left(z-p_{2}\right)\left(z-p_{3}\right)\left(z-p_{4}\right)\left(z-p_{5}\right)\left(z-p_{6}\right)}$

The numbers $K, z_{1}, \ldots, z_{6}, p_{1}, \ldots, p_{6}$ are determined by the following fuzzy rules:

Rule 1:

(R1-1): If $\operatorname{Tr}$ is about 0 , then $p_{1}$ is $0.5327+j 0.5638$.

(R1-2): If $\boldsymbol{T r}$ is about 300 , then $p_{1}$ is $0.4213+j 0.5760$.

$$
\vdots
$$

(R1-5): If $T r$ is about 992, then $p_{1}$ is $0.4807+j 0.5825$.

Rule 2:

(R2-1): If $T r$ is about 0 , then $p_{2}$ is $0.5327-j 0.5638$.

$$
\vdots
$$

Rule 13:

(R13-1): If $\operatorname{Tr}$ is about 0 , then $K$ is 0.3004 .
(R13-5): If $T r$ is about 992 , then $K$ is 0.4096 .

The symbol $T r$ represents the present track number where the actuator is at. The numbers for the poles and zeros are the ones obtained by the $H_{\infty}$ optimisation procedure. $K$ is a constant gain term in the transfer function. The fugsy variables are then defuzzified by the weighted average method. Five rules are involved for each variable, even though usually not more than two rules will be fired. This well not affect the operation since the controller scheduling process can be carried out in the back ground process. Since one always know the target track before track accessing process begins, the tuning procedure can always start first and proceed during the accessing process and convert to the target controller when the calculation is complete. Even if the conversion is not complete before reaching the target track, the original controller will still keep in action, preventing the tracking error from diverging as long as the unstable situations denoted in table. 1 are avoided.

\section{Controller Implementation}

In this section, the experimental results from the fuszy scheduling controller implementations will be presented. Applying the five operating design track data to the fuzgy rules presented in section 5 , a linear controller for all the tracks, from track 0 to track 992, on the disk can be obtained. Let $C_{n}$ denotes the controller for track number $n$ obtained by the furgy controller scheduling process, the performance of the fuzzy scheduling controller will be presented. First the controller $C_{n}$ is used to control the actuator at track $n$. Then the controller $C_{n}$ is used to control the actuator at neighboring tracks for comparison.

A TMS320C30 digital signal processor board is used for the controller implementation. The board is equipped with $4096 \times 32$ bits of ROM, $2048 \times 32$ bits of RAM. Using $33.3 \mathrm{MHz}$ clock with 16 bit $\mathrm{A} / \mathrm{D}$ and $\mathrm{D} / \mathrm{A}$ channel, the board is capable of sampling at $100 \mathrm{KHz}$; however, in this experiment only $10.43 K H z$ sampling rate and a very small amount of RAM space is used so that practical application of the method will be possible. The demodulated position error signal (PES) is fed into the controller as the error signal, and the calculated manipulated control input is send to the voice coil drive to close the loop. The controller and the tuning process are all performed in real time. While the control action is being calculated in the foreground loop, the tuning process is performed in the microprocessor as well in the background process.

Only the typical situations will be presented. Figs.5a,b shows the PES signal when $C_{500}$ is used on track number 500 and on track number 600 , and Figs.7a,b shows the power spectrum density of the signals. Even though the 
PES signal it self does not show much difference, it can be observed in the PSD plot that using $C_{500}$ on track number 500 has lower error signal intensity in the 10 to $200 \mathrm{~Hz}$ region. This indicates that the controller tuning has in fact improved the system performance. This tendency is further enhanced when the $C_{500}$ controller is used for track number 400 (Fig.7c).

\section{Conclusions}

In this paper, the fuzzy logic was used for a high performance system. A fuzzy controller scheduling capability was implemented on a computer disk file actuator track following controller. The fuzzy scheduling controller was implemented in the back ground process so that the original high bandwidth loop obtained by the traditional linear controller can be maintained. It was shown that, as the requirement for system accuracy and performance was raised, several mechanical problems appears in the design. These problem made the adequate actuator plant model varies from the inside tracks to the outside tracks on the disk. It was also shown that the variation of the model can not be described by a simple function. Therefore, the fuszy interpretation becomes the most effective tool to describe the variation.

A Zentek 3100 disk drive was used for the research. Five operation points were chosen for the design. The results showed that the performance can be improved with the controller adjusting itself. In the fuzzy tuning algorithm, the controller was expressed in pole-zero form, and groups were formed for the poles and zeros of the controllers. Five rules were used for each fuzzy variable, and a total of 65 rules were used for the 6th order controller with five design points. Simulation results showed that the resulting controller has an improved overall performance. Experimental results also showed that real time implementation of the algorithm is realistic.

\section{ACKNOWLEDGEMENT}

This work is supported by the Opto-Electronics \& Systems Lab., the Industrial Technology Research Institute, ShinChu, Taiwan. R.O.C. Special thanks to the Research and Development Group at Zentek Co., Shin-Chu, Taiwan for supplying the disk drives and the technical assistance.

\section{REFERENCES}

[1] Bajorek,C.H., 1991, "Trends in recording and control technologies and evolution of subsystem architectures for data storage," Advances in Information storage systems, edited by Bharat Bhushan, The American Society of Mechanical Engineering, pp.1-14.

[2] Bell,T.E., 1991, "Incredible shrinking computers", IEEE spectrum, May 1991, pp.37-41.
[3] Commander,R.D., and Taylor,J.R., 1980, "Servo design for an eight-inch disk file", IBM Disk Storage Technology, Feb., pp.90-98.

[4] Workman,M.L., 1987, "Digital servo control system for a data recording disk file", U.S. Patent Number: $1,679,109$, July.

[5] Franklin,G.F. Powell,J.D., Workman,M.L., 1990, Digital Control of Dynamic Systems, 2nd ed, AddisonWesley publishing Co.

[6] Harker,J.M., Brede,D.W., Pattison,R.R., Santana,G.R, Taft,L.G., 1981, " A quarter centruy of disk file innovation", IBM J. Res. Dev., Vol.25, No.5, Sep., pp.677-689.

[7] Hanselmann,H., Engelke,A., 1988, " LQG-control of a highly resonant disk drive head positioning actuator", IEEE trans. on industrial electronics, Vol.35, No.1, Feb., pp.100-104.

[8] Intel 16-bit embedded controllers, 1991, Handbook intel Co.

[9] Ljung,L. and Soderstrom,T., 1987, Theory and practice of recursive identification, The MIT Press.

[10] Mayer,J.H., 1988, "3 1/2-in. Winchesters seek slots in high-performance disktep sytems", Computer design, April 15, pp.144-150.

[11] Wood,R, 1990, "Magnetic megabits", IEEE Spectrum, May, pp.32-38.

[12] Yen,J.Y., Hallamasek,K., Horowitz,R., 1991, "Trackfollowing controller design for a compound disk drive actuator", ASME Trans. of Dyn. Sys., Mea., and Control, Vol.112, Sep., pp.391-402.

[13] Zorpette,G., 1991, " Fujitsu predicts optical-density magnetic diskn, IEEE the institute, Nov/Dec., pp.6. 

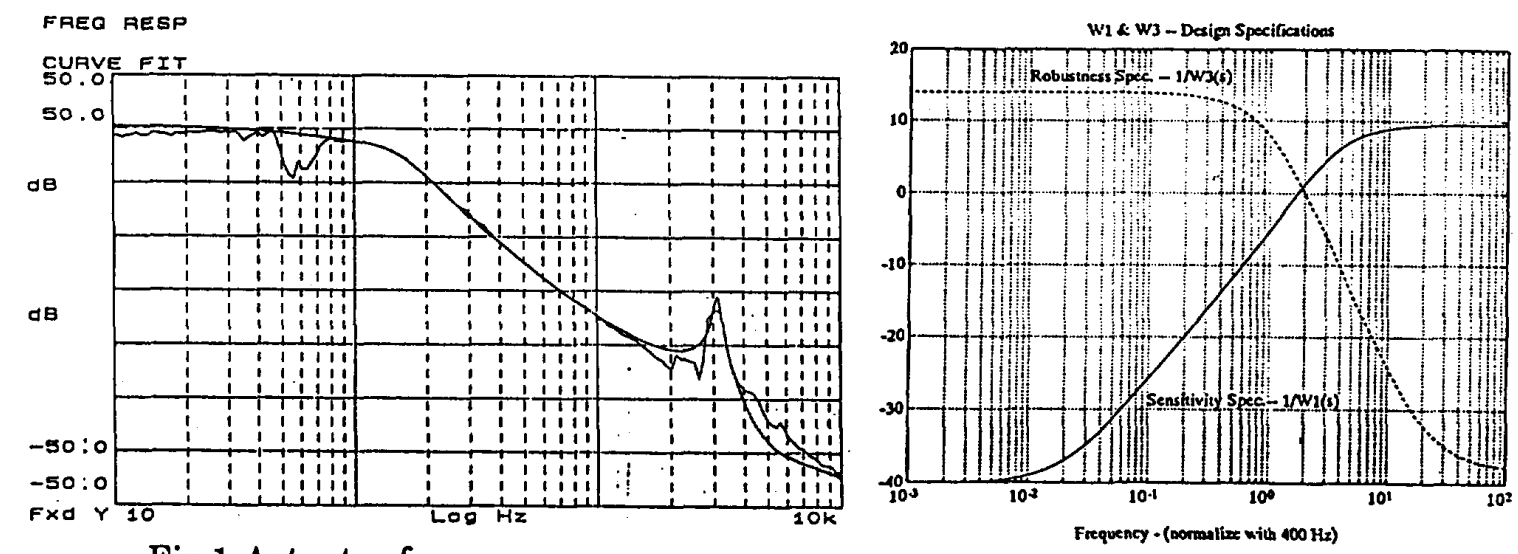

Fig.1 Actuator frequency response and least square curve fit

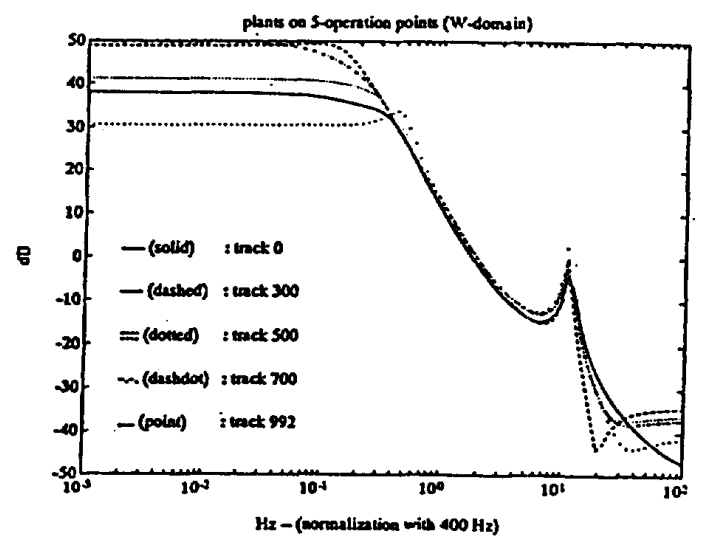

Fig.2 Actuator frequency responses on different tracks

Fig.3 Weighting functions for the $H_{\infty}$ design

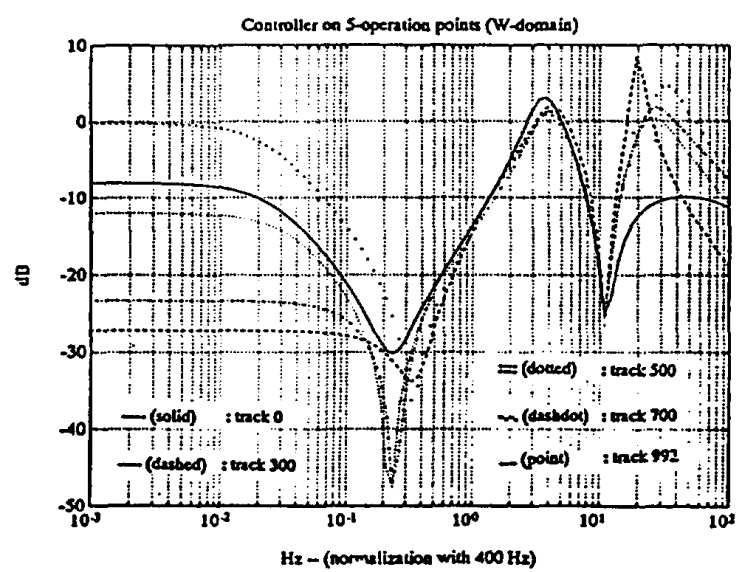

Fig.4 Frequency responses for the compensators on different tracks 


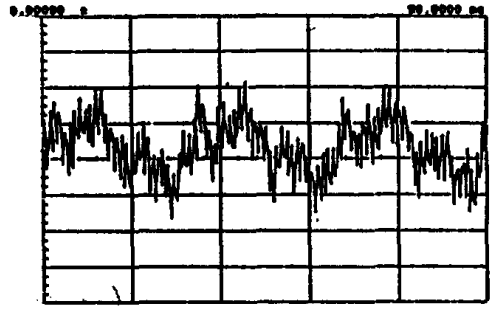

Fig.5a PES signal $C_{500} / P_{500}$ (Vertical scale $200 \mathrm{mv} / \mathrm{div}$ )

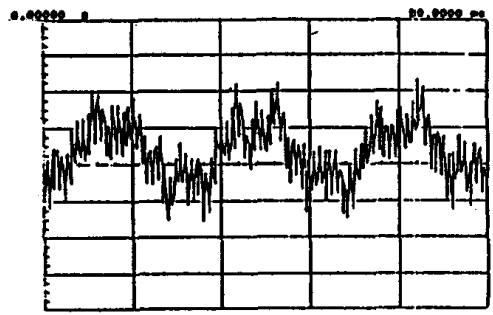

Fig.5b PES signal $C_{500} / P_{600}$ (Vertical scale $200 \mathrm{mv} /$ div)

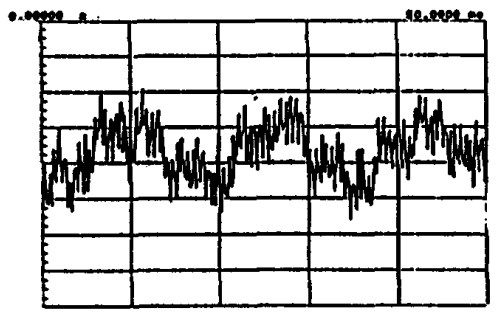

Fig.5c PES signal $C_{500} / P_{400}$ (Vertical scale $200 \mathrm{mv} /$ div)

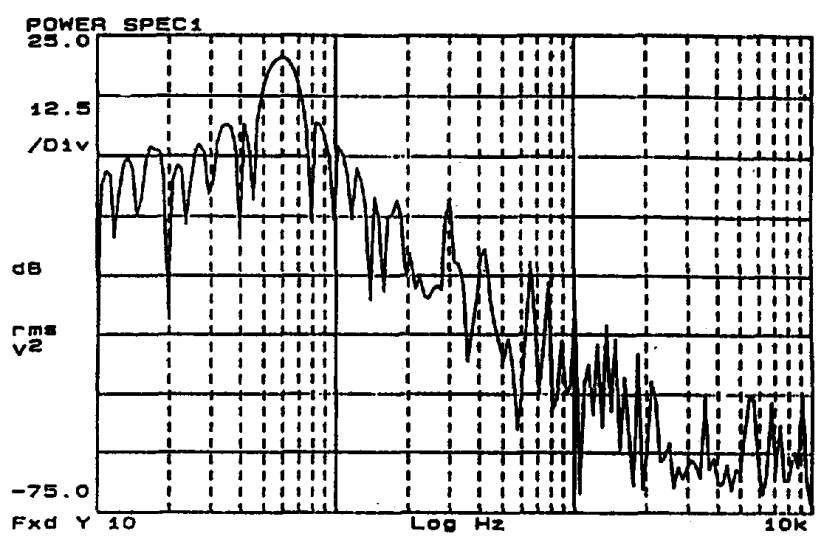

Fig.6a PSD of the PES signal $C_{500} / P_{500}$

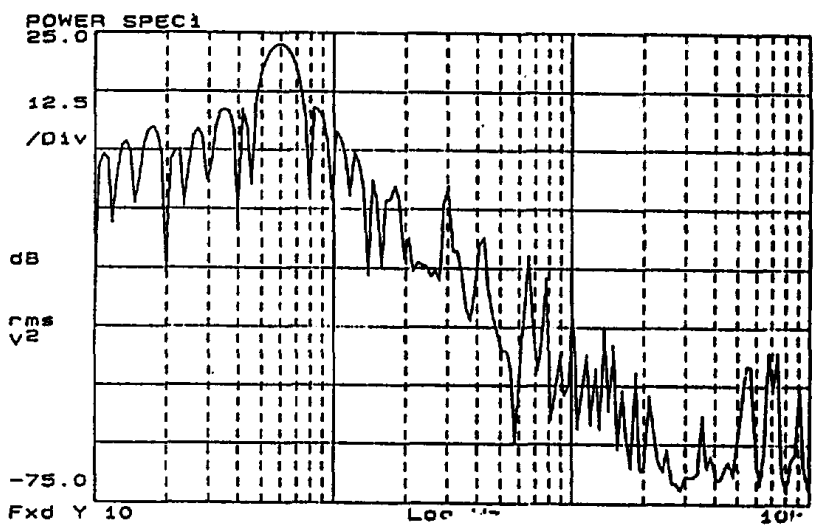

Fig.6b PSD of the PES signal $C_{500} / P_{600}$

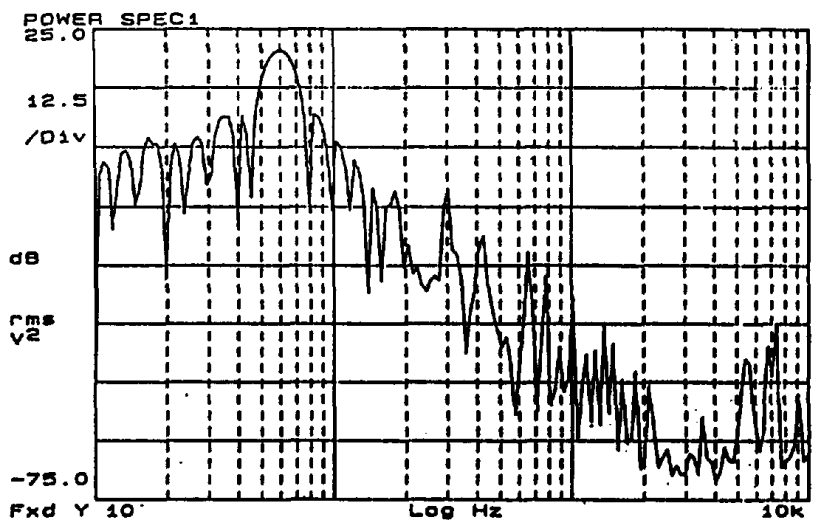

Fig.6c PSD of the PES signal $C_{500} / P_{400}$ 